\title{
DIRAC STRUCTURES ON HILBERT SPACES
}

\section{A. PARSIAN and A. SHAFEI DEH ABAD}

(Received 5 May 1997 and in revised form 4 August 1997)

\begin{abstract}
For a real Hilbert space $(H,\langle\rangle$,$) , a subspace L \subset H \oplus H$ is said to be a Dirac structure on $H$ if it is maximally isotropic with respect to the pairing $\left\langle(x, y),\left(x^{\prime}, y^{\prime}\right)\right\rangle_{+}=$ $(1 / 2)\left(\left\langle x, y^{\prime}\right\rangle+\left\langle x^{\prime}, y\right\rangle\right)$. By investigating some basic properties of these structures, it is shown that Dirac structures on $H$ are in one-to-one correspondence with isometries on $H$, and, any two Dirac structures are isometric. It is, also, proved that any Dirac structure on a smooth manifold in the sense of [1] yields a Dirac structure on some Hilbert space. The graph of any densely defined skew symmetric linear operator on a Hilbert space is, also, shown to be a Dirac structure. For a Dirac structure $L$ on $H$, every $z \in H$ is uniquely decomposed as $z=p_{1}(l)+p_{2}(l)$ for some $l \in L$, where $p_{1}$ and $p_{2}$ are projections. When $p_{1}(L)$ is closed, for any Hilbert subspace $W \subset H$, an induced Dirac structure on $W$ is introduced. The latter concept has also been generalized.
\end{abstract}

Keywords and phrases. Dirac structure, maximally isotropic, Hilbert space, $L$-admissibility.

1991 Mathematics Subject Classification. 46B20, 46C05.

1. Introduction. Dirac structures on smooth manifolds were introduced by Courant and Weinstein [1, 2] as a generalization of Poisson and presymplectic structures. The algebraic counterpart of this structure is given by Dorfman [3]. Our approach to these structures is via functional analysis. As a first step, in this paper, we study Dirac structures on Hilbert spaces. Our definition of a Dirac structure is a minor variation of the one given in [1], and the two definitions are equivalent in the finite dimensional case. Indeed, our construction includes the Dirac structures on smooth manifolds in the sense of [1] as a special case. One of the natural applications of these structures is in the area of partial differential equations. This is illustrated by giving a simple example (see Example 2.21). It is worthwhile to note that most of the techniques in finite dimension may not be applied to the infinite dimensional case. For example, the use of coordinate systems is not possible. The main properties we have employed in dealing with Dirac structures are the maximality of these structures with respect to the proposed pairing and the basic properties of Hilbert spaces.

In Section 1, we study some basic properties of Dirac structures on a real Hilbert space. This leads to some decompositions of elements in Hilbert spaces with regard to Dirac structures (see Theorem 2.10 and Corollary 2.15). As a useful application, it is shown that the graph of any densely defined skew symmetric linear operator on a Hilbert space is indeed a Dirac structure (Theorem 2.19). The interconnection of Dirac structures and certain skew symmetric linear maps is given in Theorem 2.24. Then the correspondence between the set of Dirac structures and the set of isometries on a Hilbert space is established in Theorem 2.27. In Section 3, we study the situations in which a Dirac structure on a Hilbert space, in certain sense, induces a Dirac structure 
on a Hilbert subspace. It turns out that when the subspace is admissible with respect to the Dirac structure, (see Definition 3.7), the induced Dirac structure exists (Theorem 3.6). We show that the induced Dirac structure introduced here, is consistent with the one given by Courant [1, Sec. 1.4$]$ in the finite dimensional case.

2. Dirac structures on Hilbert spaces. Let $(H,\langle\rangle$,$) be a real Hilbert space. The$ componentwise inner product on $H \oplus H$ is, also, denoted by $\langle$,$\rangle . The orthogonality on$ $H$ and $H \oplus H$ are both denoted by $\perp$. Let $\langle,\rangle_{+}$be the pairing on $H \oplus H$ defined by

$$
\left\langle(x, y),\left(x^{\prime}, y^{\prime}\right)\right\rangle_{+}=\frac{1}{2}\left(\left\langle x, y^{\prime}\right\rangle+\left\langle x^{\prime}, y\right\rangle\right) .
$$

The orthogonality with respect to $\langle,\rangle_{+}$is denoted by $\perp$.

DEFINITION 2.1. With the above notations, a Dirac structure on a Hilbert space $(H,\langle\rangle$,$) is a subspace of H \oplus H$ which is maximally isotropic under the pairing $\langle,\rangle_{+}$.

EXAMPLE 2.2. Let $A: H \rightarrow H$ be a skew symmetric linear map and let $L=\operatorname{graph}(A)$ $\subset H \oplus H$. For $(x, y),\left(x^{\prime}, y^{\prime}\right) \in L$,

$$
\begin{aligned}
\left\langle(x, y),\left(x^{\prime}, y^{\prime}\right)\right\rangle_{+} & =\left(\left\langle x, y^{\prime}\right\rangle+\left\langle x^{\prime}, y\right\rangle\right) / 2 \\
& =\left(\left\langle x, A x^{\prime}\right\rangle+\left\langle x^{\prime}, A x\right\rangle\right) / 2 \\
& =\left(\left\langle x, A x^{\prime}\right\rangle-\left\langle x, A x^{\prime}\right\rangle\right) / 2=0 .
\end{aligned}
$$

Therefore, $L$ is isotropic. Let $(a, b) \in H \oplus H$, and assume that for all $x \in H$,

$$
\langle(x, A x),(a, b)\rangle_{+}=0 .
$$

Then $\langle x, b\rangle+\langle a, A x\rangle=0$, i.e., $\langle b-A a, x\rangle=0$ for all $x \in H$. Thus, $b=A a$ and, hence, $(a, b) \in L$. This means that $L$ is a Dirac structure on $H$.

The above example is strengthened in Theorem 2.19.

LEMMA 2.3. Let $M \subset H \oplus H$ be isotropic with respect to $\langle,\rangle_{+}$. Then the closure of $M$ in $H \oplus H$ is, also, isotropic.

Proof. Assume that $(a, b) \in \bar{M}$. Take a sequence $\left(x_{n}, y_{n}\right)$ of elements of $M$ converging to $(a, b)$. Let $(x, y) \in M$, then

$$
\begin{aligned}
\langle(a, b),(x, y)\rangle_{+} & =(\langle a, y\rangle+\langle x, b\rangle) / 2 \\
& =\left(\left\langle\lim _{n \rightarrow \infty} x_{n}, y\right\rangle+\left\langle x, \lim _{n \rightarrow \infty} y_{n}\right\rangle\right) / 2 \\
& =\lim _{n \rightarrow \infty}\left(\left\langle x_{n}, y\right\rangle+\left\langle x, y_{n}\right\rangle\right) / 2 \\
& =\lim _{n \rightarrow \infty}\left\langle\left(x_{n}, y_{n}\right),(x, y)\right\rangle_{+}=0 .
\end{aligned}
$$

Similarly, for $(c, d) \in \bar{M},\langle(a, b),(c, d)\rangle_{+}=0$. Therefore, $\bar{M}$ is isotropic.

COROLlary 2.4. Every Dirac structure on $H$ is a closed subspace of $H \oplus H$ and, consequently, it is a Hilbert subspace. 
COROLlary 2.5 (Hellinger-Toeplitz theorem). Any skew symmetric linear map on a Hilbert space is continuous.

Proof. This follows from Example 2.2, Corollary 2.4 and the closed graph theorem.

LEMmA 2.6. Let $L$ be a Dirac structure on $H$ and let $p_{i}: H \oplus H \rightarrow H,(i=1,2)$, be the first and the second projections. Let $H_{1}=\{0\} \times H, H_{2}=H \times\{0\}$. Then

(i) $\operatorname{ker}\left(\left.p_{1}\right|_{L}\right)=H_{1} \cap L$,

(ii) $\operatorname{ker}\left(\left.p_{2}\right|_{L}\right)=H_{2} \cap L$,

(iii) $p_{2}\left(L \cap H_{1}\right)=p_{1}(L)^{\perp}$,

(iv) $p_{1}\left(L \cap H_{2}\right)=p_{2}(L)^{\perp}$,

(v) $\overline{p_{1}(L)}=\left(p_{2}\left(L \cap H_{1}\right)\right)^{\perp}$,

(vi) $\overline{p_{2}(L)}=\left(p_{1}\left(L \cap H_{2}\right)\right)^{\perp}$.

Proof. (i) and (ii) are clear. To prove (iii) let $y \in p_{2}\left(L \cap H_{1}\right)$ and $x \in p_{1}(L)$, then $(0, y) \in L$ and there exists $z \in H$ such that $(x, z) \in L$. Now,

$$
\langle(0, y),(x, z)\rangle_{+}=\frac{1}{2}\langle x, y\rangle=0 .
$$

Therefore, $p_{2}\left(L \cap H_{1}\right) \subset p_{1}(L)^{\perp}$. Assume that $y \in p_{1}(L)^{\perp}$. Then, for each $(x, z) \in L$, we have $\langle(0, y),(x, z)\rangle_{+}=1 / 2\langle x, y\rangle=0$. Since $L$ is maximally isotropic, $(0, y) \in L$. Therefore, $y \in p_{2}\left(L \cap H_{1}\right)$, i.e., $p_{2}\left(L \cap H_{1}\right) \subset p_{1}(L)^{\perp}$. The proof of (iv) is similar. The identities (v) and (vi) are consequences of (iii) and (iv) and the following lemma whose proof is straightforward.

LEMMA 2.7. Let $M$ be a subspace of a Hilbert space $H$, then $\bar{M}=\left(M^{\perp}\right)^{\perp}$.

Proposition 2.8. A Dirac structure L induces a skew symmetric linear form $\Omega_{1}$ on $p_{1}(L) \subset H$ defined by

$$
\Omega_{1}\left(p_{1}(x, y)\right)=x^{*}, \quad x^{*}(z)=\langle z, y\rangle \quad \text { for all } z \in p_{1}(L) .
$$

Moreover,

$$
\operatorname{ker} \Omega_{1}=p_{1}\left\{L \cap\left(p_{1}(L) \times p_{1}(L)^{\perp}\right)\right\} .
$$

A similar statement holds for $\Omega_{2}$ on $p_{2}(L)$ and

$$
\operatorname{ker} \Omega_{2}=p_{2}\left\{L \cap\left(p_{2}(L)^{\perp} \times p_{1}(L)\right)\right\} .
$$

Proof. Let $E=p_{1}(L)$ and let $E^{*}$ be the topological dual of $E$. The map $\Omega_{1}$ is well defined: Suppose that $(x, y),\left(x, y^{\prime}\right) \in L$. Then $\left(0, y-y^{\prime}\right) \in L \cap H_{1}$. Thus, by Lemma 2.6, $y-y^{\prime} \in E^{\perp}$. Assume that $z \in E$. Then $0=\left\langle z, y-y^{\prime}\right\rangle=\langle z, y\rangle-\left\langle z, y^{\prime}\right\rangle$. Therefore, $\langle z, y\rangle=\left\langle z, y^{\prime}\right\rangle$. Since $z$ is arbitrary, $\Omega_{1}\left(p_{1}(x, y)\right)=\Omega_{1}\left(p_{1}\left(x, y^{\prime}\right)\right)$. Now, we show that $\Omega_{1}$ is skew symmetric: In fact, for $(x, y),(u, v) \in L$,

$$
\begin{aligned}
& \left\langle\Omega_{1}\left(p_{1}(x, y)\right), p_{1}(u, v)\right\rangle+\left\langle\Omega_{1}\left(p_{1}(u, v)\right), p_{1}(x, y)\right\rangle \\
& =\langle u, y\rangle+\langle x, v\rangle=2\langle(x, y),(u, v)\rangle_{+}=0 .
\end{aligned}
$$


Finally,

$$
\begin{aligned}
\operatorname{ker} \Omega_{1} & =\left\{p_{1}(x, y) \mid(x, y) \in L \text { and } \Omega_{1}\left(p_{1}(x, y)\right)=0\right\} \\
& =\left\{x \mid(x, y) \in L \text { and } x^{*} \mid E=0\right\} \\
& =\left\{x \mid(x, y) \in L \text { and } y \in p_{1}(L)^{\perp}\right\} \\
& =p_{1}\left\{L \cap\left(E \times E^{\perp}\right)\right\} .
\end{aligned}
$$

The proof of the statement about $\Omega_{2}$ is similar.

LEMMA 2.9. With the above notations, if $p_{1} \pm p_{2}: L \rightarrow H$ are surjective, then they are isomorphisms of Hilbert spaces.

Proof. Let $z=(x, y) \in \operatorname{ker}\left(p_{1}+p_{2}\right)$, then $x+y=0$. Hence, $(x,-x) \in L$. Therefore, $-2\|x\|^{2}=0$, i.e., $z=0$. Thus, $p_{1}+p_{2}$ is injective. Observe that $p_{1}+p_{2}$ is norm preserving because, for $(x, y) \in L$,

$$
\|(x, y)\|^{2}=\langle(x, y),(x, y)\rangle=\|x\|^{2}+\|y\|^{2}=\langle(x+y),(x+y)\rangle=\|x+y\|^{2} .
$$

Therefore, $\|(x, y)\|=\|x+y\|$. Consequently, Banach's theorem implies that when $p_{1}+p_{2}$ is surjective, it is an isomorphism of Hilbert spaces. The proof of the statement about $p_{1}-p_{2}$ is similar.

THEOREM 2.10. The maps $p_{1} \pm p_{2}: L \longrightarrow H$ are isomorphisms of Hilbert spaces.

Proof. By Lemma 2.9, it is enough to show that $p_{1} \pm p_{2}$ are surjective. Let $z \perp\left(p_{1}+\right.$ $\left.p_{2}\right)(L)$. By the decomposition theorem in Hilbert spaces, there are $(x, y),\left(x^{\prime}, y^{\prime}\right) \in L$ such that $(z, z)=(x, y)+\left(y^{\prime}, x^{\prime}\right)$. Therefore,

$$
2\|z\|^{2}=\langle(z, z),(z, z)\rangle=\langle(z, z),(x, y)\rangle+\left\langle(z, z),\left(y^{\prime}, x^{\prime}\right)\right\rangle=0,
$$

i.e., $z=0$. Hence, $\left(p_{1}+p_{2}\right)(L)$ is dense in $H$. Now, let $\left(\left(p_{1}+p_{2}\right)\left(x_{n}\right)\right)_{n \in N}$ be a Cauchy sequence in $H$. Since $p_{1}+p_{2}$ is norm preserving, $\left(x_{n}\right)_{n \in N}$ is a Cauchy sequence in $L$ and, hence, $\left(p_{1}+p_{2}\right)(L)=H$. Similarly, $p_{1}-p_{2}$ is an isomorphism.

LEMMA 2.11. Let $(x, y) \in H \oplus H$ such that, for all $(a, b) \in L$,

$$
\langle(x, y),(a, b)\rangle_{+}=\langle(x, y),(a, b)\rangle=0 .
$$

Then $(x, y)=0$.

Proof. Assume that $\langle(y, x),(a, b)\rangle_{+}=\langle(y, x),(a, b)\rangle=0$. Then $\langle(x+y, y+x),(a$, $b)\rangle_{+}=\langle(x+y, y+x),(a, b)\rangle=0$. Let $x+y=p_{1}(l)+p_{2}(l)$ for some $l \in L$. Then, by Lemma $2.9,(x+y, y+x)=\left(p_{1}(l), p_{2}(l)\right)+\left(p_{2}(l), p_{1}(l)\right)=l+t(l)$, where $t(l)=$ $\left(p_{2}(l), p_{1}(l)\right)$. But then $0=\langle l+t(l), l\rangle_{+}=\langle t(l), l\rangle_{+}=\|l\|^{2}$, which implies that $l=0$. Therefore, $x+y=0$, and, hence, $y=-x$. Again by Lemma 2.9, $x=p_{1}\left(l^{\prime}\right)-p_{2}\left(l^{\prime}\right)$. Then $(x,-x)=l^{\prime}-p\left(l^{\prime}\right)$ and, as above, $l^{\prime}=0$. Therefore, $x=0$ and $y=0$.

The following is a consequence of Corollary 2.4 and Lemma 2.11.

COROLLARY 2.12. Let $L$ be a Dirac structure on $H$. Then

(i) If $(x, y) \perp L$, then $(x, y) \in L$. 
(ii) $L^{\perp}=t(L)$.

(iii) $L^{\perp}$ is a Dirac structure.

THEOREM 2.13. Let $L$ be a Dirac structure on a Hilbert space $H$. Then, for $\lambda \neq 0$ and $\mu \neq 0$, the linear mapping $p_{\lambda, \mu}: L \longrightarrow H$ defined by $p_{\lambda, \mu}=\lambda p_{1}+\mu p_{2}$ is a homomorphism, which is an isometry for $|\lambda|=|\mu|=1$.

Proof. Clearly, $p_{\lambda, \mu}$ is continuous. Assume that $z=(x, y) \in \operatorname{ker}\left(\lambda p_{1}+\mu p_{2}\right)$, then $\lambda x+\mu y=0$. Therefore, $(x,-(\lambda / \mu) x) \in L$ and, hence, $-2(\lambda / \mu)\|x\|^{2}=0$, i.e., $z=0$. Therefore, $p_{\lambda, \mu}$ is injective. Now, let $\lambda \mu>0$ and $v \in p_{\lambda, \mu}(L)^{\perp}$, i.e., $\langle v, \lambda x+\mu y\rangle=0$ for all $(x, y) \in L$. Since, by Lemma 2.9, $v=x+y$ for some $(x, y) \in L$, we have, $0=$ $\langle x+y, \lambda x+\mu y\rangle=\lambda\|x\|^{2}+\mu\|y\|^{2}$. Therefore, $x=y=0$ and, hence, $v=0$. For $\lambda \mu<0$, let $v=x-y$ with $(x, y) \in L$. Then $0=\langle x-y, \lambda x+\mu y\rangle=\lambda\|x\|^{2}-\mu\|y\|^{2}$. Thus, $x=$ $y=0$ and, hence, $v=0$. Therefore, $p_{\lambda, \mu}(L)$ is dense in $H$. Without loss of generality, we may assume that $|\lambda| \leq|\mu|$. Then $|\lambda|\|(x, y)\| \leq\left\|p_{\lambda, \mu}(x, y)\right\| \leq|\mu|\|(x, y)\|$. Thus, if $\left(p_{\lambda, \mu}\left(x_{n}\right)\right)_{n \in N}$ is a Cauchy sequence in $p_{\lambda, \mu}(L)$, then $\left(x_{n}\right)_{n \in N}$ is a Cauchy sequence in $L$. Therefore, the inequality above and Corollary 2.4 implies that $p_{\lambda, \mu}(L)=H$. Now, the continuity of $p_{\lambda, \mu}^{-1}$ follows from Banach's theorem.

The following can be deduced from the proof of Theorem 2.13.

Proposition 2.14. Let $L \subset H \oplus H$ be isotropic. Then,

(i) For each $\lambda, \mu \in R, \lambda \neq 0, \mu \neq 0$, the map $p_{\lambda, \mu}: L \longrightarrow H$ defined by $(x, y) \longmapsto$ $\lambda x+\mu y$ is injective.

(ii) For $L$ to be a Dirac structure, it is sufficient that, for some $\lambda, \mu \in R, \lambda \neq 0, \mu \neq$ $0, p_{\lambda, \mu}: L \longrightarrow H$ is surjective.

COROLlary 2.15. Let $A: H \rightarrow H$ be a skew symmetric linear map. Then every $h \in H$ has a unique representation $h=x+A x$ for some $x \in H$ such that

$$
\|h\|^{2}=\|x\|^{2}+\|A x\|^{2} .
$$

Proof. Let $L=\operatorname{graph}(A)$. By Example 2.2, $L$ is a Dirac structure on $H$. Thus, by Theorem 2.13, $p_{1}+p_{2}: L \longrightarrow H$ is an isometry. Hence, for some $x \in H, h=x+A x$ with the required identity on norms.

The following example explains the relation between the Dirac structures on smooth manifolds in the sense of [1] and our definition of Dirac structures on Hilbert spaces.

EXAMPLE 2.16. Let $(M, g)$ be a Riemannian manifold endowed with an almost Dirac structure $L$ as in [1]. Using the natural isomorphism $T M \longrightarrow T^{*} M$ induced by $g, L$ can be considered as a subbundle of $T M \oplus T M$. $L$ is maximal with respect to the pairing

$$
\langle(u, v)(x, y)\rangle_{+m}=\frac{1}{2}\left(g_{m}(u, y)+g_{m}(x, v)\right) .
$$

Let $p_{i}: T M \oplus T M \longrightarrow T M$ be the $i$ th projection, $i=1,2$. Clearly, $p_{i}$ is a strong bundle map. By a well-known result on bundle maps and the finite dimensional case of Theorem 2.13, $p_{1}+p_{2}: L \longrightarrow T M$ is a strong bundle isomorphism which is an isometry on each fibre. Let $H$ be the space of $L^{2}$-sections of $T M$. Then $H \oplus H$ is the space of 
$L^{2}$-sections of $T M \oplus T M$. Clearly, the space of $L^{2}$-sections of $L$ is an isotropic subspace of $H \oplus H$. We denote this space by $\Lambda$. If $p_{i \#}$ is defined by $\left(p_{i \#}(X)\right)_{m}=p_{i}\left(X_{m}\right)$, then it follows that $p_{1 \#}+p_{2 \#}: \Lambda \longrightarrow H$ is an isometric isomorphism. By Theorem $2.13, \Lambda$ is a Dirac structure on $H$.

As special cases, one can see that the graph of the symmetric (resp., Hamiltonian) operator of a presymplectic (resp., Poisson) Riemannian manifold $M$ acting on the Hilbert space of $L^{2}$-sections of $T M\left(\right.$ resp., $\left.T^{*} M\right)$ is a Dirac structure on these Hilbert spaces.

The following is an example of a Dirac structure with our definition which is not a Dirac structure in the sense of [1].

EXAMPLE 2.17. Let $H=L^{2}(R, \mu)$, where $\mu$ is the Lebesgue measure on $R$. Then $H \oplus H$ is the Hilbert space of all $L^{2}$-sections of the trivial vector bundle $p_{1}: R^{2} \rightarrow R$. Let $L$ be the singular subbundle of the vector bundle defined by

$$
L_{\lambda}= \begin{cases}R \times\{0\} & \text { for } \lambda \neq 0, \\ 0 & \text { for } \lambda=0 .\end{cases}
$$

Using Theorem 2.13, it follows that the subspace $\Lambda$ of $H \oplus H$ consisting of all $L^{2}$ sections of $L$ is a Dirac structure on $H$. But $L$ is not an almost Dirac structure on $R$ in the sense of [1].

In order to present some interesting examples of Dirac structures, we need the following well-known lemma.

LEMmA 2.18. Let $A: H \longrightarrow H$ be a densely defined skew symmetric linear map. Then $\operatorname{graph}(A)$ is closed.

Proof. Let $\left(x_{n}, A x_{n}\right)_{n \in N}$ be a sequence in graph $(A)$ converging to $(x, y)$, and let $u \in \operatorname{Dom}(A)$. Then

$$
\lim _{n \rightarrow \infty}\left(u,-A x_{n}\right)=\lim _{n \rightarrow \infty}\left(A u, x_{n}\right) .
$$

Therefore, $\langle u,-y\rangle=\langle A u, x\rangle$ and, consequently, $x \in \operatorname{Dom}\left(A^{*}\right)=\operatorname{Dom}(A)$ and $\langle u, y\rangle=$ $\langle u,-A x\rangle$. Thus, $u \perp(y+A x)$ for all $u \in \operatorname{Dom}(A)$. Since $\overline{\operatorname{Dom}(A)}=H$, we have $y+A x=0$ and, hence, $(x, y) \in \operatorname{graph}(A)$.

TheOrem 2.19. Let $A: H \rightarrow H$ be a densely defined skew symmetric linear map. Then $\operatorname{graph}(A)$ is a Dirac structure on $H$.

Proof. By Lemma 2.18, $\operatorname{graph}(A)$ is a closed subspace of $H \oplus H$. Let $\operatorname{graph}(A) \cup$ $\{(a, b)\}$ be an isotropic subset of $H \oplus H$ with $(a, b) \perp \operatorname{graph}(A)$. Then, for all $x \in$ $\operatorname{Dom}(A)$, we have,

$$
\langle a, A x\rangle+\langle b, x\rangle=\langle a, x\rangle+\langle b, A x\rangle=0 .
$$

Consequently, $\langle a+b, x+A x\rangle=0$ for all $x \in \operatorname{Dom}(A)$. Let $u=a+b$, then we have

$$
0=\langle u, x+A x\rangle=\langle u, x\rangle+\langle u, A x\rangle,
$$


i.e., $\langle x,-u\rangle=\langle A x, u\rangle$. Therefore, $u \in \operatorname{Dom}\left(A^{*}\right)=\operatorname{Dom}(A)$ and $\langle x,-u\rangle=\langle x,-A u\rangle$ for all $x \in \operatorname{Dom}(A)$. Consequently, $A u=u$. Now, we have

$$
\|u\|^{2}=\langle A u, u\rangle=\langle u,-A u\rangle=-\|u\|^{2},
$$

which implies that $a=-b$. Since graph $(A) \cup\{(a, b)\}$ is isotropic, we have $a=b=0$. This shows that the $\operatorname{graph}(A)$ is a maximally isotropic subspace of $H \oplus H$, i.e., it is a Dirac structure on $H$.

COROLlary 2.20. Let $A: H \rightarrow H$ be a densely defined skew symmetric linear map. Then the map $(I+A): \operatorname{Dom}(A) \rightarrow H$ is bijective.

Proof. This follows from Theorems 2.13 and 2.19.

Under the hypotheses of Corollary 2.20, since the map $I+A$ is bijective, the pull-back of the inner product of $H$ by this map is an inner product on $\operatorname{Dom}(A)$. We denote this inner product and the associated norm by $\langle,\rangle_{p}$ and \|\|$_{p}$, respectively. Then $\operatorname{Dom}(A)$ is a Hilbert space with this inner product. Since, for every $x \in \operatorname{Dom}(A)$,

$$
\|x\|_{p}^{2}=\|x+A x\|^{2}=\|x\|^{2}+\|A x\|^{2} \geq\|A x\|^{2},
$$

the linear operator $A: \operatorname{Dom}(A) \longrightarrow H$ is a contracting operator.

EXAMPLE 2.21. Let $H=L^{2}(R)$ and let $A=d / d x$ be the differential operator defined on the subspace $C_{0}^{1}(R) \subset H$, the space of continuously differentiable real functions with compact support. Using integration by parts, it can be seen that $A$ is skew symmetric. Thus, by Theorem 2.19, graph $(A)$ is a Dirac structure on $H$.

Now, we study the properties of left and right Dirac structures.

Definition 2.22. A Dirac structure $L$ on $H$ is said to be a left (resp., right) Dirac structure if $p_{1}(L)$ (resp., $\left.p_{2}(L)\right)$ is closed. The mapping $t: H \oplus H \longrightarrow H \oplus H$, defined by $t(x, y)=(y, x)$, changes any left Dirac structures to a right one.

Let $L$ be a Dirac structure on $H$ and $L_{1}=\{(0, y) \in L\}=H_{1} \cap L$, with the notation of Lemma 2.6. Clearly, $L_{1}$ is a closed subspace of $L$. Let $L_{2}=\left(L_{1}\right)^{\perp}$ be the orthocomplement of $L_{1}$ in $L$. Clearly, $L_{2}$ is a closed subspace of $L$.

LEMMA 2.23. With the above notations, $p_{2}\left(L_{2}\right) \subset \overline{p_{1}(L)}$.

PRoof. Let $z \in p_{1}(L)^{\perp}=p_{2}\left(L \cap H_{1}\right)$ and $w \in p_{2}\left(L_{2}\right)$. Then there exists $y \in p_{1}(L)$ such that $(y, w) \in L_{2}$. On the other hand, $(0, z) \in L_{1}$. Since $L_{1} \perp L_{2}$ in $L$,

$$
0=\langle(y, w),(0, z)\rangle=\langle w, z\rangle
$$

Therefore, $z \perp w$. Since $z \in p_{1}(L)^{\perp}$ is arbitrary, $w \perp p_{1}(L)^{\perp}$, i.e., $w \in\left(p_{1}(L)^{\perp}\right)^{\perp}=\overline{p_{1}(L)}$. Consequently, $p_{2}\left(L_{2}\right) \subset \overline{p_{1}(L)}$.

With the notations above, the mapping $p_{1}: L_{2} \longrightarrow p_{1}(L)$ is bijective. We denote the inverse map by $q_{1}: p_{1}(L) \longrightarrow L_{2}$. Let $\Omega: p_{1}(L) \longrightarrow \overline{p_{1}(L)}$ be defined by $\Omega=p_{2} \circ q_{1}$ which is well-defined by the previous lemma. Clearly, $\Omega$ is a skew symmetric linear map. Conversely, let $E$ be a closed subspace of $H$ and let $\Omega: E \rightarrow E$ be a skew symmetric 
linear map. Let $L_{2}=\operatorname{graph}(\Omega)$ and $L_{1}=\{0\} \times E^{\perp}$. Clearly, $L_{1}$ is a closed subspace of $H \oplus$ $H$ and $L_{1} \perp L_{2}$. Let $L=L_{1} \oplus L_{2}$. Then $L$ is an isotropic subspace of $H \oplus H$. To see this, let $(x, y) \in H \oplus H$ such that $\{(x, y)\} \cup L$ is isotropic. Then, for $z \in E^{\perp},\langle(x, y),(0, z)\rangle_{+}=$ $(1 / 2)\langle x, z\rangle=0$. Therefore, $x \in E$ and $(x, \Omega(x)) \in L$. Consequently, $(0, y-\Omega(x)) \perp L$. Therefore, $y-\Omega(x) \in E^{\perp}$ and $L$ is a Dirac structure with $p_{1}(L)=E$.

The above discussion can be summarized in the following theorem.

THEOREM 2.24. Let $H$ be a Hilbert space. Then

(i) For any Dirac structure $L$ on $H$, there corresponds a skew symmetric linear map $\Omega: p_{1}(L) \longrightarrow \overline{p_{1}(L)}$ such that $L$ is the direct sum of $\operatorname{graph}(\Omega)$ and the vector space $L_{1}=\left\{(0, y) \mid y \in p_{1}(L)^{\perp}\right\}$. Moreover, $\operatorname{graph}(\Omega) \perp L_{1}$.

(ii) Conversely, let $E$ be a closed subspace of $H$ and let $\Omega: E \rightarrow E$ be a skew symmetric linear map. Then $\operatorname{graph}(\Omega) \oplus\left\{(0, y) \mid y \in E^{\perp}\right\}$ is a Dirac structure on $H$ and $\operatorname{graph}(\Omega) \perp\left\{(0, y) \mid y \in E^{\perp}\right\}$.

Let $L$ be a Dirac structure on $H$. As we have seen earlier, the mappings $p_{1} \pm p_{2}: L \longrightarrow$ $H$ are isometries. Thus, the map $A: H \rightarrow H$, defined by $A=\left(p_{1}-p_{2}\right) \circ\left(p_{1}+p_{2}\right)^{-1}$, is an isometry. We claim that

$$
L=\left\{\left(\left(\frac{I+A}{2}\right)(x),\left(\frac{I-A}{2}\right)(x)\right) \mid x \in H\right\} .
$$

Because $x \in H$, there exists $(z, w) \in L$ such that $x=z+w$. Then

$$
\left(\frac{I+A}{2}(x), \frac{I-A}{2}(x)\right)=\left(\frac{z+w+z-w}{2}, \frac{z+w-z+w}{2}\right)=(z, w) .
$$

On the other hand, for every $(z, w) \in L,\left(\frac{I+A}{2}(z+w), \frac{I-A}{2}(z+w)\right)=(x, y)$. The converse of the above observation is, also, true in the following sense.

Proposition 2.25. Let $A: H \rightarrow H$ be a surjective linear isometry. Then the vector space $L_{A} \subset H \oplus H$ is defined by

$$
L_{A}=\left\{\left(\frac{I+A}{2}(x), \frac{I-A}{2}(x)\right) \mid x \in H\right\}
$$

is a Dirac structure on $H$.

Proof. Let $x \in H$. Then

$$
\begin{aligned}
\left\langle\left(\frac{I+A}{2}(x), \frac{I-A}{2}(x)\right),\right. & \left.\left(\frac{I+A}{2}(y), \frac{I-A}{2}(y)\right)\right\rangle_{+} \\
& =\frac{1}{8}\langle(I+A)(x),(I-A)(y)\rangle+\frac{1}{8}\langle(I-A)(x),(I+A)(x)\rangle \\
& =\frac{1}{4}(\langle x, y\rangle-\langle A x, A y\rangle)=0 .
\end{aligned}
$$

Therefore, $L_{A}$ is isotropic under the pairing $\langle,\rangle_{+}$. Let $(z, w) \in H \oplus H$ such that $L_{A} \cup$ $\{(z, w)\}$ is isotropic. Then, for each $x \in H, 0=\left\langle(z, w),\left(\frac{I+A}{2} x, \frac{I-A}{2} x\right)\right\rangle_{+}=\left(\left\langle z, \frac{I-A}{2} x\right\rangle+\right.$ $\left.\left\langle\frac{I+A}{2} x, w\right\rangle\right) / 2$, i.e., $\langle z, x-A x\rangle+\langle x+A x, w\rangle=0$. Therefore, $\langle z, x\rangle-\langle z, A x\rangle+\langle x, w\rangle+$ $\langle A x, w\rangle=0$ and, hence, $\langle z+w, x\rangle+\langle A x, w-z\rangle=0$. Since $A$ is a surjective isometry, $\langle A(z+w), A x\rangle+\langle A x, w-z\rangle=0$, i.e., $z-w=A(z+w)$. Therefore, $(z, w)=\left(\frac{I+A}{2}(z+\right.$ $\left.w), \frac{I-A}{2}(z+w)\right)$. Thus, $L_{A}$ is a Dirac structure. 
LEMмA 2.26. Let $A, B: H \rightarrow H$ be two surjective linear isometries on the Hilbert space H. If $L_{A}=L_{B}$, then $A=B$.

Proof. Assume that $L_{A}=L_{B}$ and let $x, y \in H$. Since $L_{A}$ is isotropic,

$$
\langle((I+A) x,(I-A) x),((I+B) y,(I-B) y)\rangle_{+}=0 .
$$

Hence,

$$
\langle(I+A) x,(I-B) y\rangle+\langle(I+B) y,(I-A) x\rangle=0 .
$$

Consequently, $\langle(I-B) *(I+A) x, y\rangle+\left\langle(I+B)^{*}(I-A) x, y\right\rangle=0$. Thus, $(I-B) *(I+A)+$ $(I+B)^{*}(I-A)=0$. This implies that $B^{*} A=I$. Since $A$ and $B$ are surjective isometries, it follows that $A=B$.

Proposition 2.25 and Lemma 2.26 can be summarized in the following.

THEOREM 2.27. The set of Dirac structures on a Hilbert space $H$ is in one-to-one correspondence with the set of isometries on $\mathrm{H}$.

Proposition 2.28. Let $E \subset H$ be a subspace. Then $L=E \times E^{\perp}$ is a Dirac structure on $H$ if and only if $E$ is closed.

Proof. Clearly, $L$ is isotropic. Let $E$ be closed and let $(a, b) \in H \oplus H$ such that for all $(x, y) \in L,\langle(x, y),(a, b)\rangle_{+}=0$. Observe that $(x, 0) \in L$ and $(0, y) \in L$ because, $x \in E$ and $y \in E^{\perp}$. Thus, $\langle a, y\rangle=0$ and $\langle b, x\rangle=0$, i.e., $a \in E$ and $b \in E^{\perp}$. Conversely, let $L=$ $E \times E^{\perp}$ be a Dirac structure on $H$. Let $c \in \bar{E}-E$ and $b \in E^{\perp}$. Since $L=E \times E^{\perp}=E \times(\bar{E})^{\perp}$, we have $(c, b) \perp(x, y)$. Now, the maximality of $L$ implies that $(c, b) \in L=E \times E^{\perp}$, i.e., $c \in E$. Therefore, $E$ is closed.

COROLLARY 2.29. Let $H$ be a Hilbert space.

(i) Let $L$ be a Dirac structure on $H$ and assume that $p_{2}(L)=p_{1}(L)^{\perp}$. Then $L=$ $p_{1}(L) \times p_{2}(L)$.

(ii) Let $E$ and $F$ be two closed subspaces of $H$ such that $L=E \times F$ is a Dirac structure on $H$. Then $E^{\perp}=F$ and $F^{\perp}=E$.

(iii) Let $E$ and $F$ be two subspaces of $H$ and let $L=E \times F$ be a Dirac structure on $H$. Then $L$ is both a left and a right Dirac structure on $H$.

REMARK 2.30. Let $E \subset H$ be a Hilbert subspace and let $M$ be a dense subspace of $E$. Let $\Omega: E \rightarrow E$ be a skew symmetric linear map and assume that $L$ is the Dirac structure on $H$ induced by $\Omega$. Set

$$
L_{M}=\{(x, \Omega(x)) \mid x \in M\} \oplus\left\{(0, y) \mid y \in M^{\perp}\right\} .
$$

Similar to the proof of Theorem 2.24, it can be seen that $L=\overline{L_{M}}$.

REMARK 2.31. As we have seen earlier, for any Dirac structure $L$ on a Hilbert space $H$, the map $p_{1}+p_{2}: L \longrightarrow H$ is a bijective linear isometry. Thus, for any two Dirac structures $L_{i},(i=1,2)$, on $H$, there are bijective linear isometries $\phi_{1}: L_{1} \longrightarrow L_{2}$ and $\phi_{2}: L_{1}^{\perp} \longrightarrow L_{2}^{\perp}$. Therefore, there exists a bijective linear isometry $\phi: H \oplus H=L_{1} \oplus L_{1}^{\perp} \longrightarrow$ $L_{2} \oplus L_{2}^{\perp}=H \oplus H$ defined by $\phi(x, y)=\left(\phi_{1}(x), \phi_{2}(y)\right)$ such that $\phi\left(L_{1}\right)=L_{2}$. 
REMARK 2.32. Since a Dirac structure $L$ is isotropic under the pairing $\langle,\rangle_{+}$, it does not intersect with any subspace of $H \oplus H$ which is definite under the pairing $\langle,\rangle_{+}$. Let $P=\{(x+y, y+x) \mid(x, y) \in L\}, N=\{(x-y, y-x) \mid(x, y) \in L\}$. Then, as subspaces of $H \oplus H, P$ is maximal positive definite and $N$ is maximal negative definite under the above pairing. Clearly, $L$ is the graph of the map $A_{L}: N \rightarrow P$ defined by $A_{L}(x-y, y-$ $x)=(x+y, y+x)$.

The following proposition deals with Dirac structures on two Hilbert spaces and the proof is straightforward.

Proposition 2.33. Let $(H,\langle\rangle$,$) and (G,\langle\rangle$,$) be two Hilbert spaces.$

(i) Let $L$ be a Dirac structure on $H$ and let $\phi: H \longrightarrow G$ be a surjective linear isometry. Then $K=\{(\phi(x), \phi(y)) \mid(x, y) \in L\}$ is a Dirac structure on $G$.

(ii) Let $L$ and $M$ be Dirac structures on $H$ and $G$, respectively. Then $L \oplus M$ is a Dirac structure on $H \oplus G$.

3. Induced Dirac structures. Let $(H,\langle\rangle$,$) be a real Hilbert space and let H=W \oplus V$, where $W$ and $V$ are closed subspaces of $H$. For $x \in H$ and $E \subset H$, the components of $x$ and $E$ in $W$ are denoted by $x_{W}$ and $E_{W}$, respectively. Let $L$ be a left Dirac structure on $H$. Consider the space

$$
L(W)=\left\{(x, y) \in W \times W \mid \exists z \in H \text { such that }(x, z) \in L \text { and } z_{W}=y\right\} .
$$

As in Section 1 , the projections on $L \rightarrow H$ are denoted by $p_{1}$ and $p_{2}$. Let $W_{1}=W \cap p_{1}(L)$ and let $W_{2}$ be the orthocomplement of $W_{1}$ in $W$. We define

$$
L_{W}=L(W)+\left(\{0\} \times W_{2}\right) .
$$

Clearly, $L_{W}$ is isotropic with respect to $\langle,\rangle_{+}$. We show that it is indeed maximally isotropic in $W \oplus W$. Let $(a, b) \in(W \times W) \cap\left(L_{W}\right)^{\perp}$. Then, for $y \in W_{2},\langle a, y\rangle=0$. Hence, $a \in W_{2}^{\perp} \cap W=W_{1}$ since $W_{1}$ is closed. Consequently, there exists $t \in W$ such that $(a, t) \in L(W)$. It follows that $(0, b-t) \in\left(L_{W}\right)^{\perp}$. This implies that $b-t \in W_{2}$. Thus, $(a, b)=(a, t)+(0, b-t) \in L_{W}$.

Therefore, we have proven the following.

THEOREM 3.1. Let $L$ be a left Dirac structure on $H$ and let $W$ be a closed subspace of $H$. Then $L_{W}$ is a left Dirac structure on $W$.

DEFINITION 3.2. With the above notations, $L_{W}$ is called the induced left Dirac structure on $W$.

REMARK 3.3. Following the notations above, let $x \in p_{1}(L)^{\perp}$, then $(0, x) \in L$. Since $H=W \oplus W^{\perp}, x=x_{W}+x_{W^{\perp}}$. Observing that $W=W_{1} \oplus W_{2}$, we have, $x=x_{W W_{1}}+x_{W W_{2}}+$ $x_{W^{\perp}}$ while $x_{W W_{1}} \in W_{1}, x_{W W_{2}} \in W_{2}$, with the expected meaning. Therefore, there exists $z \in W$ such that $\left(x_{W W_{1}}, z\right) \in L_{W}$. Now, we have

$$
0=\left\langle\left(0, x_{W}\right),\left(x_{W W_{1}}, z\right)\right\rangle_{+}=\left\langle x_{W}, x_{W W_{1}}\right\rangle=\left\|x_{W W_{1}}\right\|^{2}+\left\langle x_{W W_{2}}, x_{W W_{1}}\right\rangle=\left\|x_{W W_{1}}\right\|^{2} .
$$

Thus, $x_{W W_{1}}=0$, i.e., $\left(p_{1}(L)^{\perp}\right)_{W} \subset W_{2}$. Let $z \in W_{2}$ and let $z \perp\left(p_{1}(L)^{\perp}\right)_{W}$. Then, for every $y \in p_{1}(L)^{\perp}$, 


$$
\langle z, y\rangle=\left\langle z, y_{W_{2}}+y_{W_{2}}\right\rangle=0 .
$$

Therefore, $z \in W_{1} \cap W_{2}$. Thus, $z=0$, i.e., $\left(p_{1}(L)^{\perp}\right)_{W}$ is dense in $W_{2}$. Consequently, in the finite dimensional case, $\{0\} \times W_{2}=\{0\} \times\left(p_{1}(L)^{\perp}\right)_{W} \subset L(W)$. Therefore, $L_{W}=$ $L(W)+\left\{(x, y) \in W \times W \mid \exists z \in H\right.$ such that $(x, z) \in L$ and $\left.z_{W}=y\right\}$ as proved in [1, Sec. 1.4].

In the following, we introduce the induced structures on some special subspaces of real Hilbert spaces for Dirac structures which are not necessarily left or right. Nevertheless, this generalizes the induced left Dirac structures.

DEFinITION 3.4. Let $L$ be a Dirac structure on $H$ and let $W \subset H$ be a closed subspace of $H$. We say that $W$ is $L$-admissible if $p_{\lambda, \mu}\left(L_{W}\right)=W$, for some $\lambda \neq 0$ and $\mu \neq 0$, where $p_{\lambda, \mu}=\lambda p_{1}+\mu p_{2}$, and $L_{W} \subset W \oplus W$ is the same as introduced above. Let $\overline{L_{W}}$ be the closure of $L_{W}$ in $W \oplus W$.

THEOREM 3.5. Let $L$ be a Dirac structure on $H$ and let $W$ be an $L$-admissible subspace of $H$ with some $\lambda=\mu$ and some $\lambda=-\mu$. Assume that $(u, v) \in\left(\overline{L_{W}}\right)^{\perp} \cap\left(\overline{L_{W}}\right) \perp$, where the orthocomplements are taken in $W \oplus W$. Then $(u, v)=0$.

Proof. Suppose that $x \in W$ and $(x, z) \in L$. Then $\left(x, z_{W}\right) \in \overline{L_{W}}$ and

$$
\left\langle(u, v),\left(x, z_{W}\right)\right\rangle_{+}=\left\langle(u, v),\left(x, z_{W}\right)\right\rangle=0 .
$$

Hence,

$$
\langle(u, v),(x, z)\rangle_{+}=\left\langle(u, v),\left(x, z_{W}\right)\right\rangle_{+}+\left\langle(u, v),\left(0, z_{W^{\perp}}\right)\right\rangle_{+}=0
$$

and

$$
\langle(u, v),(x, z)\rangle=\left\langle(u, v),\left(x, z_{W}\right)\right\rangle+\left\langle(u, v),\left(0, z_{W^{\perp}}\right)\right\rangle=0 .
$$

Therefore,

$$
\langle u+v, x+z\rangle=2\langle(u, v),(x, z)\rangle_{+}+\langle(u, v),(x, z)\rangle=0 .
$$

Thus, $(u+v) \perp W$, i.e., $u=-v$ and, hence, $\langle u, x-z\rangle=0$ for all $(x, z) \in L$. Consequently, by Theorem 2.10, $u \perp W$ and, hence $u=0, v=0$.

THEOREM 3.6. Let $L$ be a Dirac structure on $H$ and let $W$ be an $L$-admissible subspace of $H$ with some $\lambda=\mu$ and some $\lambda=-\mu$. Then, $\overline{L_{W}}$ is a Dirac structure on $W$.

Proof. Clearly, $\overline{L_{W}}$ is an isotropic subspace of $W \oplus W$. Let $(a, b) \in W \oplus W$ such that $(a, b) \in\left(\overline{L_{W}}\right) \perp$. By the decomposition theorem in Hilbert spaces, we have, $(a, b)=$ $(s, t)+(u, v)$, with $(s, t) \in\left(\overline{L_{W}}\right)$ and $(u, v) \in\left(\overline{L_{W}}\right)^{\perp}$. But $\overline{L_{W}} \subset\left(\overline{L_{W}}\right) \perp$ and $(u, v) \in$ $\left(\overline{L_{W}}\right)^{\perp} \cap\left(\overline{L_{W}}\right)^{\perp}$. Therefore, $(u, v)=0$ and, hence, $(a, b) \in \bar{L}_{W}$.

Let $W$ be an $L$-admissible subspace of $H$ for some $\lambda$ and $\mu$. Since the restriction map $p_{\lambda, \mu}: L_{W} \longrightarrow W$ is a homeomorphism, $L_{W}$ is a closed subspace of $W \oplus W$. Therefore, Proposition 2.14 implies that $L_{W}$ is a Dirac structure on $W$. This leads to the following definition. 
DeFinITION 3.7. Let $L$ be a Dirac structure on $H$ and let $W$ be an $L$-admissible subspace of $H$. Then, $L_{W}$ is called the induced left-type Dirac structure on $W$.

REMARK 3.8. The induced left-type Dirac structure is transitive. Let $V$ be an $L$ admissible subspace of $H$ and let $W$ be an $L_{V}$-admissible subspace of $V$. Let $L_{V W}$ be the induced left-type Dirac structure of $L_{V}$ to $W$. Then the decomposition theorem of Hilbert spaces asserts that $W$ is an $L$-admissible subspace of $H$ and $L_{V W}=L_{W}$. Consequently, the induced left-type Dirac structures (from $L$ and $L_{V}$ ) to $W$ are identical.

REMARK 3.9. If $L$ is a left Dirac structure on $H$ and $W \subset H$ is a Hilbert subspace, then $W$ is $L$-admissible. This is a consequence of Proposition 2.14. Therefore, the concept of induced left Dirac structure is a particular case of the notion of induced left-type Dirac structure on $L$-admissible Hilbert subspaces.

ACKNOWLEDGEMENT. The authors thank Professor Rahim Zaare-Nahandi for his help with this paper. Also, they would like to thank the referee for some very useful comments.

\section{REFERENCES}

[1] T. J. Courant, Dirac manifolds, Trans. Amer. Math. Soc. 319 (1990), no. 2, 631-661. MR 90m:58065. Zbl 850.70212.

[2] T. J. Courant and A. Weinstein, Beyond Poisson structures, Action hamiltoniennes de groupes. Troisieme theoreme de Lie (Paris), Travaux en Cours, vol. 27, Hermann, 1988, Semin. Sud-Rhodan. Géom. VIII, pp. 39-49. MR 89h:58054. Zbl 698.58020.

[3] I. Dorfman, Dirac structures and integrability of nonlinear evolution equations, Nonlinear Science: Theory and Applications, John Wiley \& Sons, Ltd., Chichester, 1993. MR 94j:58081.

[4] N. Dunford and J. T. Schwartz, Linear Operators. I. General Theory, With the assistance of W. G. Bade and R. G. Bartle. Pure and Applied Mathematics, vol. 7, Interscience Publishers, Inc., New York, 1958. MR 22\#8302. Zbl 084.10402.

Parsian: Institute for Studies in Theoretical Physics and Mathematics, P.O. Box 19395-1745, TEHRAN, IRAN

Abad: Department of Mathematics, University of Tehran, P.O. Box 14155-6455, TEHRAN, IRAN 


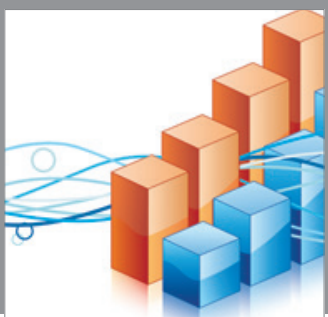

Advances in

Operations Research

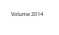

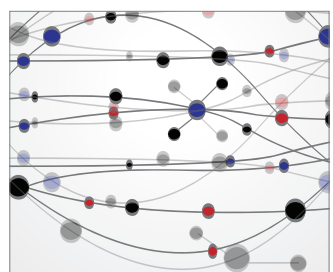

\section{The Scientific} World Journal
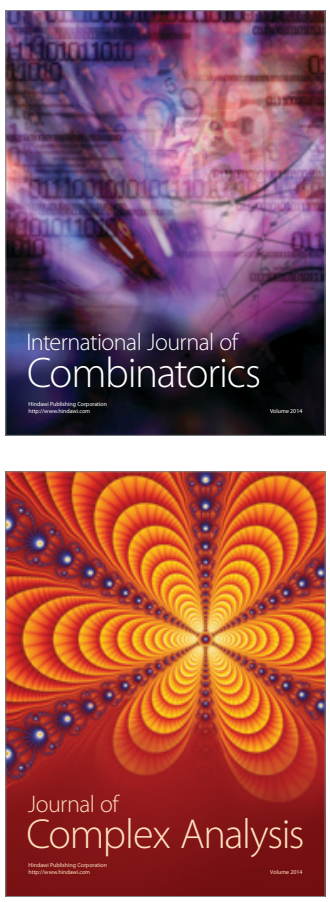

International Journal of

Mathematics and

Mathematical

Sciences
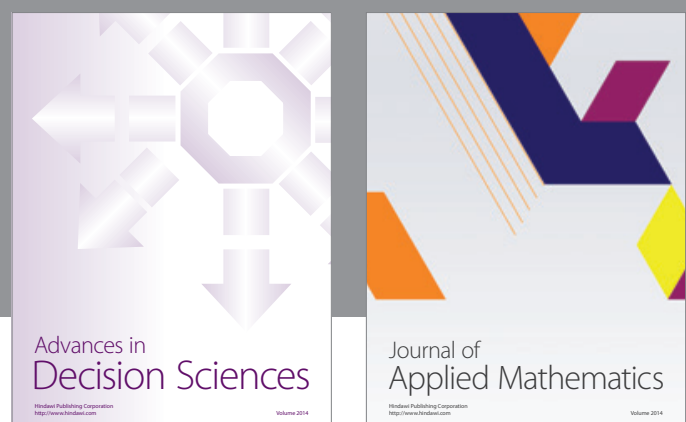

Journal of

Applied Mathematics
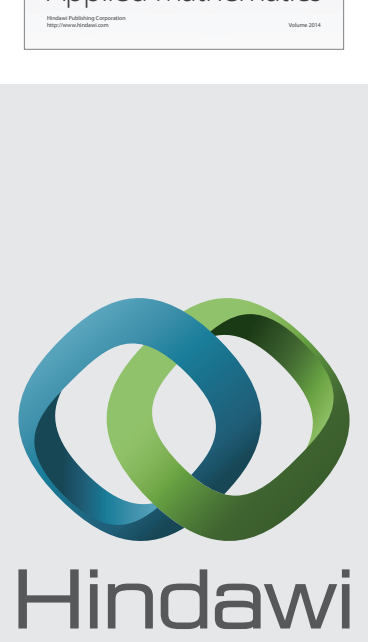

Submit your manuscripts at http://www.hindawi.com
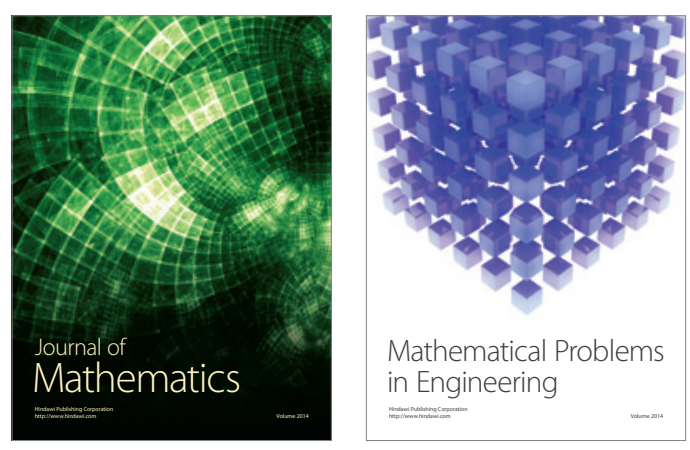

Mathematical Problems in Engineering
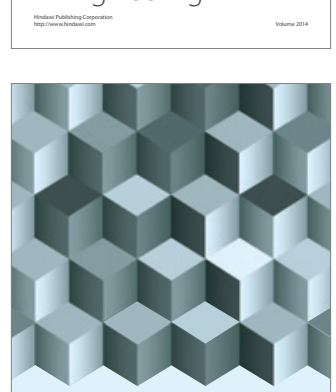

Journal of

Function Spaces
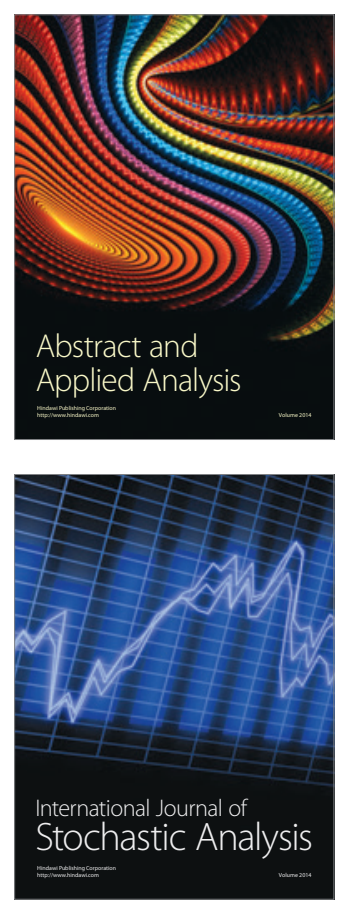

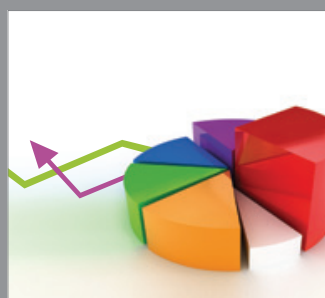

ournal of

Probability and Statistics

Promensencen
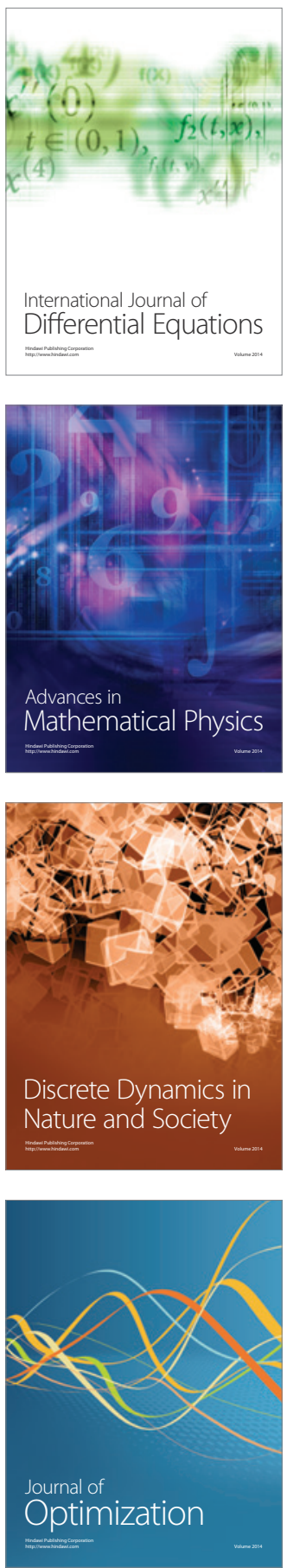